\title{
Exosomes from Thymic Stromal Lymphopoietin- Activated Dendritic Cells Promote Th2 Differentiation through the OX40 Ligand
}

\author{
Li Huang Xinxing Zhang Meijuan Wang Zhengrong Chen Yongdong Yan \\ Wenjing Gu Jiahong Tan Wujun Jiang Wei Ji \\ Department of Pediatric Pulmonology, Children's Hospital of Soochow University, Suzhou, China
}

\section{Keywords}

Thymic stromal lymphopoietin · Dendritic cells · Exosomes . OX40 ligand $\cdot$ T-helper cells

\begin{abstract}
Objectives: Exosomes are extracellular vesicles released from various inflammatory cells, such as T cells, B cells, dendritic cells (DCs), and mast cells, which have been implicated in the modulation of immune response in asthma. This study aimed to investigate whether exosomes from DCs activated by thymic stromal lymphopoietin (TSLP) play a role in T-helper cell differentiation through the OX40 ligand (OX40L). Methods: Serum samples from patients with asthma were collected to measure the levels of OX40L, T-helper type 1 (Th1) cytokine interferon (IFN)- $\gamma$, and T-helper type 2 (Th2) cytokine interleukin (IL)-4 by enzyme-linked immunosorbent assay (ELISA). Exosomes were isolated from TSLP-activated DCs and co-cultured with CD4+ T cells. Western blot and ELISA assays were used to measure the levels of OX40L, IFN- $\gamma$, and IL-4 in DCs and CD4+ T cells. Flow cytometry was applied to detect Th1 and Th2 cells. Results: OX40L and IL-4 were increased and IFN- $\gamma$ was decreased in serum from asthmatic patients compared with healthy controls. TSLP in-
\end{abstract}

duced DCs to express OX40L in released exosomes, which could promote proliferation of CD4+ T cells, elevate the level of IL-4, and promote Th2 differentiation. Conclusion: Blockade of OX40L in DC-derived exosomes could inhibit exosome-mediated CD4+ T proliferation and Th2 differentiation.

(c) 2018 S. Karger AG, Basel

\section{Introduction}

Asthma is a common chronic inflammatory respiratory disorder of the lower airways and characteristic of recurrent airway obstruction around the world. Asthma can be classified as mild, moderate, or severe according to the National Asthma Education and Prevention Program, Global Initiative for Asthma (GINA) [1]. Inflammatory cells, including CD4+ and CD8+ T lymphocytes, and dendritic cells (DCs) are known to modulate airway inflammation and immune response in asthma [2]. The

L.H. and X.Z. share first authorship and contributed equally to this work.

\section{KARGER}

(c) 2018 S. Karger AG, Basel

E-Mail karger@karger.com

www.karger.com/pat 
major induction factor of airway inflammation and asthma is the imbalance between T-helper type 1 (Th1) and T-helper type 2 (Th2) immune responses [3]. DCs are antigen-presenting cells (APCs) of the mammalian immune system which can initiate asthma via presenting antigens to $\mathrm{T}$ cells to determine the type of immune response by activating naive $T$ cells [4].

Thymic stromal lymphopoietin (TSLP) is a cytokine released by airway epithelial cells in response to allergens $[5,6]$. It stimulates the differentiation of immature DCs into mature DCs, which migrate to the draining lymph nodes [7]. It is reported that TSLP can activate DCs to prime naive $\mathrm{CD} 4+\mathrm{T}$ cells to differentiate into Th2 through OX40L, the ligand of OX40, which interacts with OX40 expressed in activated $\mathrm{T}$ cells and contributes to the balance in asthma $[8,9]$. OX40L can be generated by airway smooth muscles, epithelial cells, mast cells, and DCs to regulate Th2 responses [10]. The TSLP-OX40L signaling pathway mediated by Th2 responses is involved in inflammation and the immune system $[11,12]$. Therefore, the mechanism of DCs, TSLP, and OX40L provided a direction for our research in asthma and airway inflammation.

Exosomes have been studied in association with the pathogenesis of asthma [13]. Various inflammatory cells implicated in asthmatic processes including $B$ and $T$ lymphocytes, DCs, mast cells, and epithelial cells can release exosomes [13]. Exosomes are small vesicles $(30-100 \mathrm{~nm})$ that enable cellular communication by shuttling different molecules such as nucleic acids, lipids, and proteins. Exosomes released by DCs contain molecules and are able to specifically stimulate T-cell responses [14]. Thus, this study hopes to explore the effect of exosomes derived from TSLP-activated DCs in the proliferation and differentiation of CD4+ T cells.

\section{Materials and Methods}

\section{Serum Samples}

Serum samples were obtained from 15 patients with severe asthma and 16 patients with mild and moderate asthma; 18 healthy persons served as normal controls. All participants signed informed consent and this study was approved by the ethics committee of the Children's Hospital of Soochow University.

\section{Cell Sorting of DCs and CD4+ T Cells}

Peripheral blood samples from healthy controls were used to isolate peripheral blood mononuclear cells (PBMCs). For the PBMC preparations, DCs and CD4+ T cells were sorted and purified by anti-CD31 and anti-CD4 microbead isolation kits (Miltenyi Biotec, Auburn, CA, USA), respectively, according to the manufacturer's protocol. After cell sorting, the purity of the isolated cell populations was evaluated by the FACS Canto II flow cytometer (Becton Dickinson Biosciences, San Diego, CA, USA) and, acquiring a representative number of sorted cells, it was consistently greater than $90 \%$.

\section{Exosome Isolation and Identification}

Exosomes were prepared from the supernatant of DC cultures. Briefly, DCs were cultured at $1 \times 10^{6}$ cells $/ \mathrm{mL}$ in RPMI 1640 (Gibco, USA) and stimulated with PBS or rhTSLP (15ng/ml; R\&D Systems, Minneapolis, MN, USA). The culture medium supernatants were collected to isolate exosomes using ultracentrifugation by the Optima L-100XP Ultracentrifuge (Beckman Coulter, USA) as previously described [15]. The purified exosomes were re-suspended in PBS and stored at $-80^{\circ} \mathrm{C}$. Exosomes were also observed directly under a transmission electron microscope. The protein concentration was determined using the Bradford dye assay. To confirm the purified pellets as exosomes, Western blot was performed with anti-human CD63 (1:1,000; Abcam, Cambridge, UK).

\section{Co-Culture of Exosomes and CD4+ T Cells}

CD4+ T cells $\left(1 \times 10^{6}\right)$ were incubated in 6 -well plates with 1 $\mu \mathrm{g}$ of total protein of TSLP-activated DC-derived exosomes (DEXs) with or without treatment of $10 \mu \mathrm{g} / \mathrm{mL}$ anti-humanOX40L mAb. The proliferation of CD4+ T cells was quantified by tritiated thymidine $\left({ }^{3} \mathrm{H}-\mathrm{TdR}\right)$ incorporation (PerkinElmer Life Sciences, Vienna, Austria) according to the manufacturer's protocol.

\section{ELISA Measurement of OX40L, IFN- $\gamma$, and IL-4}

Quantitative analysis of TNF- $\alpha$, IFN- $\gamma$, and IL- 4 in serum samples and cell culture supernatants was performed by enzymelinked immunosorbent assay (ELISA) procedures using Quantikine ELISA kits (R\&D Systems) according to the manufacturer's instructions. Each sample was tested in triplicate.

\section{Western Blot}

The proteins were extracted from exosomes by RIPA lysis buffer, electrophoresed with SDS/PAGE, and transferred into the PVDF membrane. After blocking, the blots were incubated with primary antibodies against CD63 (1:1,000; Abcam), OX40L (1: 1,000; Santa Cruz Biotechnology, Santa Cruz, CA, USA), and $\beta$-actin (1:10,000; Proteintech, Wuhan, China) at $4{ }^{\circ} \mathrm{C}$ followed by incubation with horseradish peroxidase-conjugated goat anti-rabbit secondary antibodies (1:200; Abcam). Enhanced chemiluminescence reagent (Thermo Scientific, Shanghai, China) was used to visualize the protein bands.

\section{Flow Cytometric Analysis}

CD4+ T cells co-cultured with DEXs were subjected to analysis of differentiation of T cells. CD4+ T cells were collected, washed twice with PBS, and stained with FITC anti-CD4 (BioLegend, San Diego, CA, USA) for 20 min under room temperature. Followed by fixation and permeabilization, intracellular cytokines IFN- $\gamma$ and IL- 4 were stained with PE anti-IFN- $\gamma$ (BioLegend) and PE anti-IL-4 (BioLegend), respectively. Flow cytometry (Cytomics FC500; Beckman Coulter) was performed to identify Th1 and Th2 cells. 


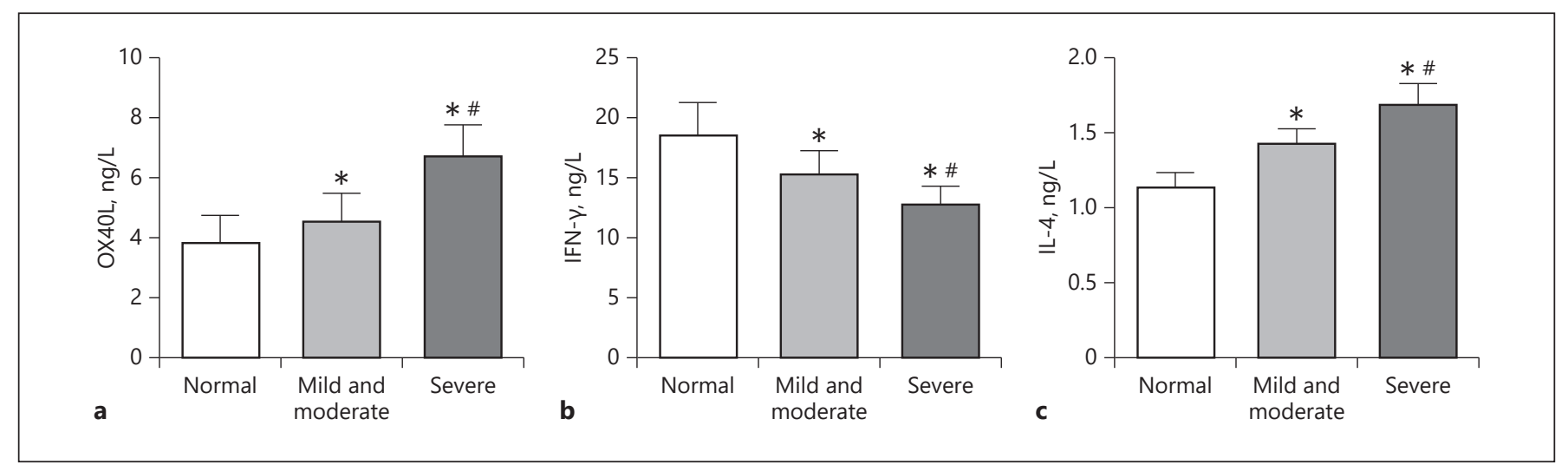

Fig. 1. Association of OX40L, IFN- $\gamma$, and IL-4 in serum of asthmatic patients. a-c The level of OX40L, IFN- $\gamma$, and IL-4 in serum patients with mild and moderate and severe asthma and healthy controls. ${ }^{*} p<0.05$ vs. normal group; ${ }^{\#} \mathrm{p}<0.05$ vs. mild and moderate group.

\section{Statistical Analysis}

All data were analyzed with GraphPad Prism version 5.0 software (GraphPad Software, San Diego, CA, USA) and expressed as mean \pm standard deviation (SD). Statistical differences between the two groups were assessed using two-tailed Student's $t$ test. All experiments were repeated at least three times. A $p$ value $<0.05$ was considered significant.

\section{Results}

Association of OX40L, IFN- $\gamma$, and IL-4 in Serum of Asthmatic Patients

Using ELISA assay, we examined the levels of OX40L, Th1 cytokine IFN- $\gamma$, and Th2 cytokine IL- 4 in serum from asthmatic patients and healthy controls. The results showed that OX40L (Fig. 1a) and IL-4 (Fig. 1c) in patients with severe asthma were increased in comparison with patients with mild and moderate asthma or healthy controls $(p<0.05)$, while OX40L and IL-4 in patients with mild and moderate asthma were increased compared with healthy controls $(p<0.05)$. On the contrary, IFN- $\gamma$ (Fig. 1b) was decreased in patients with asthma compared with healthy controls $(p<0.05)$. These results suggested that OX40L was associated with IFN- $\gamma$ and IL-4 in asthma.

\section{TSLP Induced DCs to Release Exosomes and Express OX40L}

TSLP is a cytokine released by airway epithelial cells in response to bacterial allergens in asthma and it can activate DCs [7]. As shown in Figure 2a and b, OX40L and soluble OX40L (sOX40L) were significantly increased in

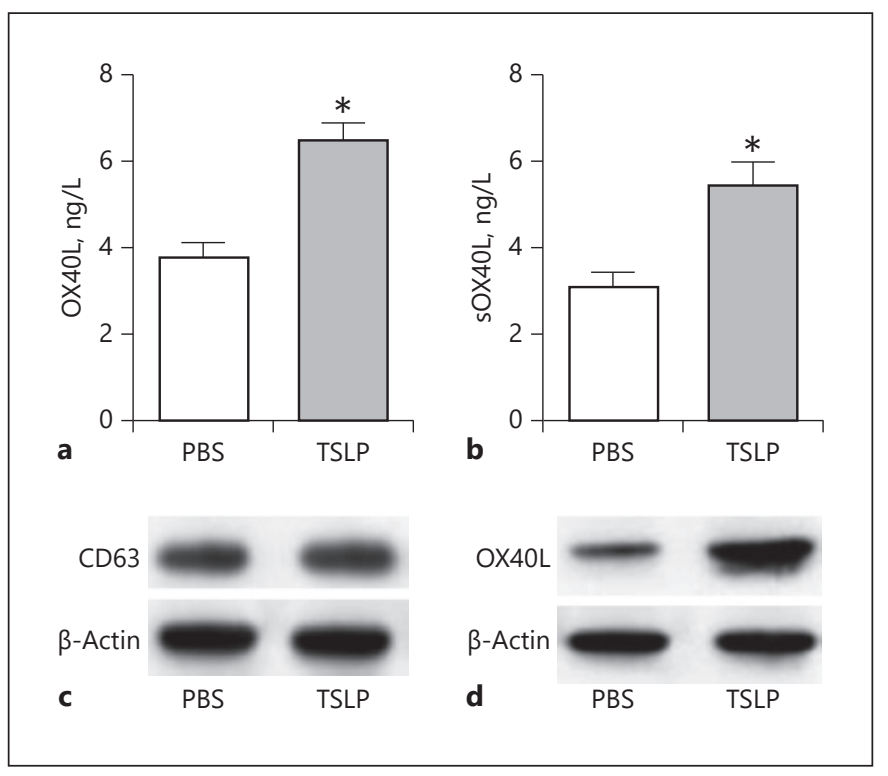

Fig. 2. TSLP induced DCs to release exosomes and express OX40L. a, b The levels of OX40L and soluble OX40L (sOX40L) in culture supernatant of TSLP-stimulated DCs were detected by ELISA. c, d CD63 and OX40L in DC-derived exosomes were detected by Western blot. ${ }^{*} p<0.05$ vs. PBS group.

culture supernatants of TSLP-stimulated DCs. In order to explore the effects of TSLP-activated DC exosomes on $\mathrm{CD} 4+\mathrm{T}$ cells, the exosomes were isolated by successive centrifugation. Transmission electron microscopy was conducted to evaluate exosome morphology (online suppl. data; for all online suppl. material, see www.karger. com/doi/10.1159/000493013). The exosomes derived 

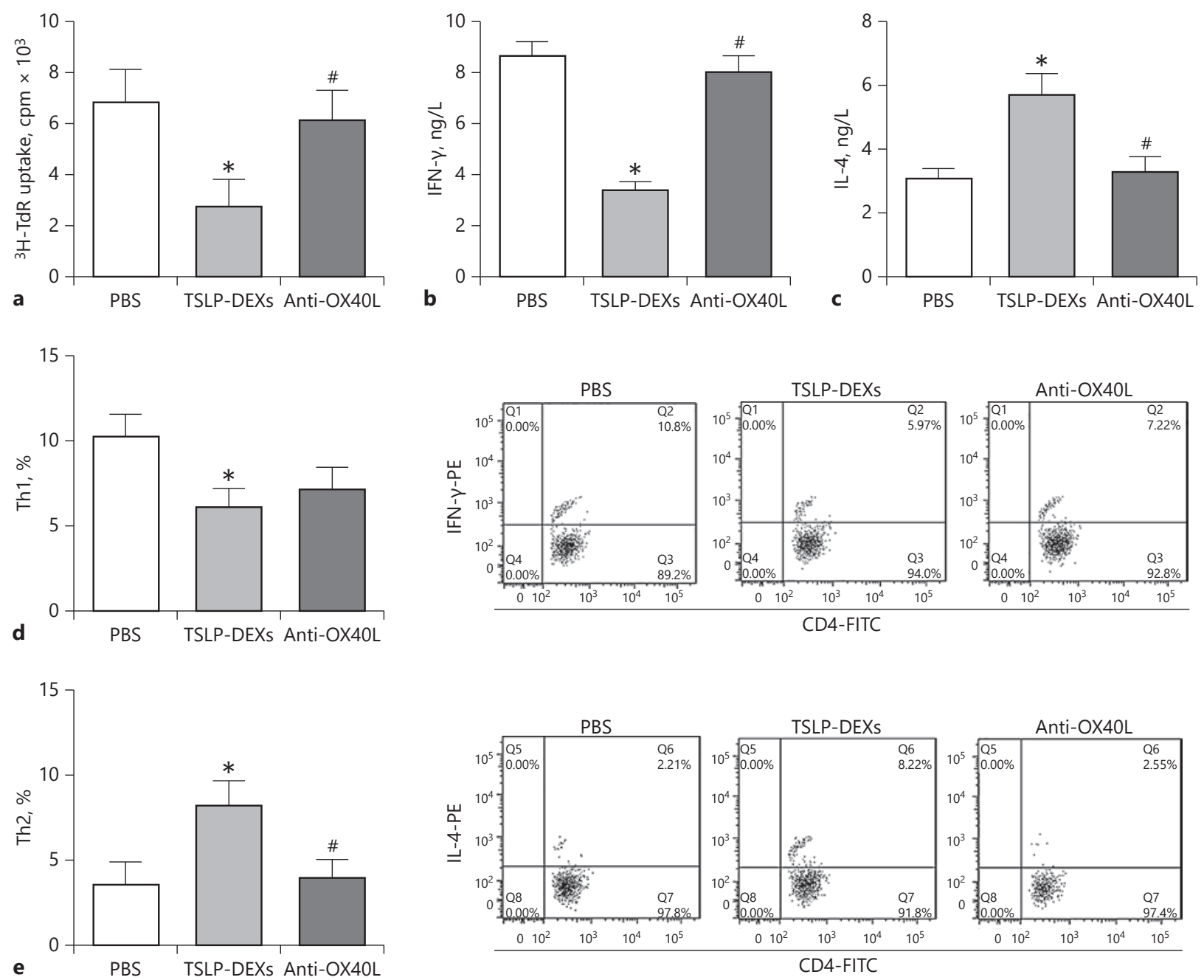

Fig. 3. Exosomes from TSLP-induced DCs promote Th2 differentiation through OX40L. a CD4+ T cells were co-incubated with TSLP-induced DC-derived exosomes (TSLP-DEXs) in the treatment with or without antiOX40L mAb. The proliferation of CD4+ T cells was quantified by ${ }^{3} \mathrm{H}-\mathrm{TdR}$. b, c The levels of IFN- $\gamma$ and IL-4 in CD4+ T cells were detected by ELISA. d, e The number of Th1 and Th2 cells in CD4+ T cells was determined by flow cytometry. ${ }^{*} p<0.05$ vs. PBS group; ${ }^{*} p<0.05$ vs. TSLP-DEXs group.

from DCs were pleomorphic with sizes varying from 30 to $120 \mathrm{~nm}$ and the protein concentration was approximately $0.1 \mu \mathrm{g} / \mu \mathrm{L}$ in $500 \mu \mathrm{L}$. Western blot indicated that exosomes expressed CD63, a marker of membrane structure (Fig. 2c). We also found that OX40L was expressed and higher in TSLP-activated DCs (Fig. 2d), suggesting that DCs constitutively released exosomes and expressed OX40L.

\section{Exosomes from TSLP-Induced DCs Promote Th2} Differentiation through $O X 40 \mathrm{~L}$

To investigate the effects of exosomes derived from TSLP-activated DCs on the proliferation and differentiation of T cells, CD4+ T cells were co-incubated with DEXs in the treatment with or without anti-OX40L mAb for 72 $\mathrm{h}$ in plates pre-coated with anti-CD3 and anti-CD28 $\mathrm{mAb}$. Compared with the PBS control without exosomes in culture medium, CD4+ T cells in the TSLP-DEXs group showed a lower proliferation rate, which was in- 
creased by anti-OX40L mAb (Fig. 3a). In the following experiments, we found that IFN- $\gamma$ (Fig. 3b) was decreased while IL-4 (Fig. 3c) was increased in the TSLP-DEXs group compared with the control group, and this was reversed in the anti-OX40L group. In addition, the number of Th1 cells (CD4+IFN- $\gamma+)$ was reduced while the number of Th2 cells (CD4+IL-4+) was increased in the TSLPDEXs group compared with the control group (Fig. 3d, e). Treatment with exosomes and anti-OX40L mAb completely inhibited the differentiation of Th2 cells to the level in the control group (Fig. 3e). These results demonstrated that OX40L in exosomes derived from TSLP-activated $\mathrm{DCs}$ promotes $\mathrm{CD} 4+\mathrm{T}$-cell differentiation into Th2 cells.

\section{Discussion}

DCs are specialized effector cells and the most important and primary APCs in the immune system. They take up the antigen, process and present it to T lymphocytes, and thereby contribute to innate immunity [16]. Based on the different types of antigens and cytokines, DCs can regulate T-lymphocyte polarization $[17,18]$. The interaction of DCs with T lymphocytes is initiated by MHC class I/II at the surface of DC specificity to the TCR of T lymphocytes, and further supported by the interaction between secondary signals such as OX40L-OX40, CD80/86-CD28, and ICAM-1-LFA-1 [19]. With completion of the molecular circuit with DCs, T lymphocytes polarize towards Th1, Th2, Th17, or regulatory T cells (Tregs). Many studies have shown that TSLP can stimulate DCs to prime naive CD4+ T cells to differentiate into Th2 type [20, 21].

In recent years, exosomes that contain a wide variety of molecules have been recognized as players in intercellular communication and immune response. In relation to the pathobiology of asthma, exosomes are released from mast cells, eosinophils, DCs, T cells, and airway epithelial cells. These in turn can trigger the activation or repression of other asthma-associated cells and enhance allergic responses [22]. In asthma, exosomes affect the inflammatory microenvironment which ultimately determines the development or alleviation of the pathological symptoms. For example, mast cell-derived exosomes induce DCs to have an antigen-presenting capacity for $\mathrm{T}$ cells and modulate the activation of T lymphocytes to secrete cytokines such as IL-6, IL-12, and IFN- $\gamma[23,24]$. Exosomes are also released by eosinophils and increased in asthmatic patients; they contain the main eosinophilic

DC-Derived Exosomes in Th2 Differentiation proteins and may play a role in driving the progression of asthma via eosinophils by increasing the production of chemokines, reactive oxygen species (ROS), and nitric oxide (NO) $[25,26]$. B-cell-derived exosomes can specifically present antigens to $\mathrm{T}$ cells and induce $\mathrm{T}$-cell responses [27]. Like other immune cells, T lymphocytes can also release exosomes [28-30]. Exosomes released by activated CD4+ $\mathrm{T}$ cells contain many proteins including CD4+ T cell markers such as CD4, TCR, and CD25, as well as liposomal-associated membrane protein 1 (LAMP1) and lymphocyte function-associated antigen-1 (LFA1 ), and suppress the immunity by CD8+ T lymphocytes [28].

DEXs contribute to allergic inflammation as well. These exosomes, like their parent cells, are able to present allergens and stimulate $\mathrm{T}$-cell responses by direct contact with CD4+ T cells or via the capture of these exosomes by other APCs [31, 32]. In this study, we found that TSLP activated DCs to release exosomes which can induce the production of Th2 cytokines such as IL-4 and promote Th2 differentiation. However, the mechanism involving components of exosomes release into the culture and the impact on T cells cannot be excluded. Based on previous studies, interaction with OX40L and OX40 facilitated CD4+ T cells to differentiate to Th2 cells [33]. In addition, Li et al. [34] found that bone marrow-derived mast cells constitutively released exosomes that expressed OX40L, which partially promoted Th2 cell differentiation. In our study, TSLP induced DCs to express OX40L. Therefore, we used anti-OX40L mAb to block OX40L on DEXs and found that anti-OX40L mAb significantly decreased their ability to promote the differentiation of Th2 cells. This demonstrated that OX40L was the molecular mechanism by which DEXs promoted the differentiation of Th2 cells. DEXs contain several molecules such as HLA-DR, MHC class I and II, CD63, CD86, and CD54 on their surface [35]. Possibly, these surface molecules or other intraluminal components of DEXs also participate in the regulatory process. Furthermore, this study limitation relates to the experimental conditions in vitro and the result may not completely represent the conditions in vivo. Therefore, in vivo studies are needed to elucidate the effects of DEXs on CD4+ T cells.

In conclusion, we found that exosomes derived from TSLP-activated DCs promoted the proliferation of CD4+ $\mathrm{T}$ cells and differentiation of Th2 cells through OX40L. This represents a novel mechanism of cell-to-cell communication. These findings may be important for future exosome engineering to create exosome-based vaccines for immunotherapy. 


\section{Statement of Ethics}

All participants signed informed consent. This study was approved by the ethics committee of the Children's Hospital of Soochow University.

\section{Disclosure Statement}

The authors declare no commercial or financial conflicts of interest.

\section{Funding Sources}

This work was supported by Suzhou Science and Technology Projects (grant No. SYS201558 to L.H.), the Jiangsu Provincial Commission of Health and Family Planning project (grant No. H201622 to L.H.), and the National Natural Science Foundation of China (grant No. 81570016 to W.J. and grant No. 81401296 to Z.C.).

\section{References}

1 Bousquet J: Global initiative for asthma (GINA) and its objectives. Clin Exp Allergy. 2000 Jun;30 Suppl 1:2-5.

2 Hamid Q, Tulic' MK, Liu MC, Moqbel R. Inflammatory cells in asthma: mechanisms and implications for therapy. J Allergy Clin Immunol. 2003 Jan;111(1 Suppl):S5-S12.

3 Kim Y, Lee S, Kim YS, Lawler S, Gho YS, Kim YK, et al. Regulation of Th1/Th2 cells in asthma development: a mathematical model. Math Biosci Eng. 2013 Aug;10(4):1095-133.

4 Yamane H, Paul WE. Early signaling events that underlie fate decisions of naive CD4(+) T cells toward distinct $\mathrm{T}$-helper cell subsets. Immunol Rev. 2013 Mar;252(1):12-23.

5 Kato A, Favoreto S Jr, Avila PC, Schleimer RP. TLR3- and Th2 cytokine-dependent production of thymic stromal lymphopoietin in human airway epithelial cells. J Immunol. 2007 Jul;179(2):1080-7.

6 Lee HC, Headley MB, Loo YM, Berlin A, Gale M Jr, Debley JS, et al. Thymic stromal lymphopoietin is induced by respiratory syncytial virus-infected airway epithelial cells and promotes a type 2 response to infection. J Allergy Clin Immunol. 2012 Nov;130(5):1187-96.

7 Melum GR, Farkas L, Scheel C, Van Dieren B, Gran E, Liu YJ, et al. A thymic stromal lymphopoietin-responsive dendritic cell subset mediates allergic responses in the upper airway mucosa. J Allergy Clin Immunol. 2014 Sep;134(3):613-621.e7.

8 Wang YH, Ito T, Wang YH, Homey B, Watanabe N, Martin R, et al. Maintenance and polarization of human $\mathrm{TH} 2$ central memory $\mathrm{T}$ cells by thymic stromal lymphopoietin-activated dendritic cells. Immunity. 2006 Jun; 24(6):827-38

9 Ito T, Wang YH, Duramad O, Hori T, Delespesse GJ, Watanabe N, et al. TSLP-activated dendritic cells induce an inflammatory $\mathrm{T}$ helper type 2 cell response through OX40 ligand. J Exp Med. 2005 Nov;202(9):1213-23.

10 Kaur D, Brightling C. OX40/OX40 ligand interactions in $\mathrm{T}$-cell regulation and asthma. Chest. 2012 Feb;141(2):494-9.
11 Li DQ, Zhang L, Pflugfelder SC, De Paiva CS, Zhang X, Zhao G, et al. Short ragweed pollen triggers allergic inflammation through Tolllike receptor 4-dependent thymic stromal lymphopoietin/OX40 ligand/OX40 signaling pathways. J Allergy Clin Immunol. 2011 Dec; 128(6):1318-1325.e2.

12 Meng Q, Liu X, Li P, He L, Xie J, Gao X, et al. The influence of house dust mite sublingual immunotherapy on the TSLP-OX40L signaling pathway in patients with allergic rhinitis. Int Forum Allergy Rhinol. 2016 Aug;6(8): 862-70.

13 Sastre B, Cañas JA, Rodrigo-Muñoz JM, Del Pozo V. Novel Modulators of Asthma and Allergy: exosomes and MicroRNAs. Front Immunol. 2017 Jul;8:826.

14 Théry C, Duban L, Segura E, Véron P, Lantz $\mathrm{O}$, Amigorena $\mathrm{S}$. Indirect activation of naïve CD4+ T cells by dendritic cell-derived exosomes. Nat Immunol. 2002 Dec;3(12):115662.

15 Théry C, Amigorena S, Raposo G, Clayton A Isolation and characterization of exosomes from cell culture supernatants and biological fluids. Curr Protoc Cell Biol.2006 Apr;Chapter 3:Unit 3.22

16 Gaurav R, Agrawal DK. Clinical view on the importance of dendritic cells in asthma. Expert Rev Clin Immunol. 2013 Oct;9(10):899919.

17 Walsh KP, Mills KH. Dendritic cells and other innate determinants of T helper cell polarisation. Trends Immunol. 2013 Nov;34(11): 521-30.

18 Hivroz C, Chemin K, Tourret M, Bohineust A. Crosstalk between T lymphocytes and dendritic cells. Crit Rev Immunol. 2012;32(2): 139-55.

19 Lombardi V, Singh AK, Akbari O. The role of costimulatory molecules in allergic disease and asthma. Int Arch Allergy Immunol. 2010; 151(3):179-89.

20 Kopecka J, Rozkova D, Sediva A. Plasmacytoid DCs, exposed to TSLP in synergy with TLR ligands, acquire significant potential towards Th2 polarization. Med Sci Monit Basic Res. 2013 Dec;19:291-9.
21 Zhang Y, Zhou X, Zhou B. DC-derived TSLP promotes Th2 polarization in LPS-primed allergic airway inflammation. Eur J Immunol. 2012 Jul;42(7):1735-43.

22 Fujita Y, Yoshioka Y, Ito S, Araya J, Kuwano $\mathrm{K}$, Ochiya T. Intercellular communication by extracellular vesicles and their microRNAs in asthma. Clin Ther. 2014 Jun;36(6):873-81.

23 Carroll-Portillo A, Cannon JL, te Riet J, Holmes A, Kawakami Y, Kawakami T, et al. Mast cells and dendritic cells form synapses that facilitate antigen transfer for T cell activation. J Cell Biol. 2015 Aug;210(5):851-64.

24 Dudeck A, Suender CA, Kostka SL, von Stebut E, Maurer M. Mast cells promote Th1 and Th17 responses by modulating dendritic cell maturation and function. Eur J Immunol. 2011 Jul;41(7):1883-93.

25 Mazzeo C, Cañas JA, Zafra MP, Rojas Marco A, Fernández-Nieto M, Sanz V, et al. Exosome secretion by eosinophils: A possible role in asthma pathogenesis. J Allergy Clin Immunol. 2015 Jun;135(6):1603-13.

26 Cañas JA, Sastre B, Mazzeo C, Fernández-Nieto M, Rodrigo-Muñoz JM, González-Guerra A, et al. Exosomes from eosinophils autoregulate and promote eosinophil functions. J Leukoc Biol. 2017 May;101(5):1191-9.

27 Admyre C, Bohle B, Johansson SM, FockeTejkl M, Valenta R, Scheynius A, et al. B cellderived exosomes can present allergen peptides and activate allergen-specific $\mathrm{T}$ cells to proliferate and produce $\mathrm{TH} 2$-like cytokines. J Allergy Clin Immunol. 2007 Dec;120(6): 1418-24.

28 Wahlgren J, Karlson TL, Glader P, Telemo E, Valadi H. Activated human T cells secrete exosomes that participate in IL-2 mediated immune response signaling. PLoS One. 2012; 7(11):e49723.

29 Nazimek K, Ptak W, Nowak B, Ptak M, Askenase PW, Bryniarski K. Macrophages play an essential role in antigen-specific immune suppression mediated by $\mathrm{T} \mathrm{CD}{ }^{+}$cell-derived exosomes. Immunology. 2015 Sep;146(1):2332. 
30 Yu X, Huang C, Song B, Xiao Y, Fang M, Feng $\mathrm{J}$, et al. CD4+CD25+ regulatory $\mathrm{T}$ cells-derived exosomes prolonged kidney allograft survival in a rat model. Cell Immunol. 2013 Sep-Oct;285(1-2):62-8.

31 Naslund TI, Gehrmann U, Qazi KR, Karlsson MC, Gabrielsson S. Dendritic cell-derived exosomes need to activate both $\mathrm{T}$ and $\mathrm{B}$ cells to induce antitumor immunity. J Immunol. 2013 Mar; 190:2712-9.
32 Tkach M, Kowal J, et al. Qualitative differences in T-cell activation by dendritic cell-derived extracellular vesicle subtypes. EMBO J. 2017 Oct;36:3012-28.

33 Ohshima Y, Yang LP, Uchiyama T, Tanaka Y, Baum P, Sergerie M, et al. OX40 costimulation enhances interleukin-4 (IL-4) expression at priming and promotes the differentiation of naive human $\mathrm{CD} 4(+) \mathrm{T}$ cells into high IL4-producing effectors. Blood. 1998 Nov; 92(9):3338-45.
34 Li F, Wang Y, Lin L, Wang J, Xiao H, Li J, et al. Mast Cell-Derived Exosomes Promote Th2 Cell Differentiation via OX40L-OX40 Ligation. J Immunol Res. 2016;2016:3623898.

35 Admyre C, Grunewald J, Thyberg J, Gripenbäck S, Tornling G, Eklund A, et al. Exosomes with major histocompatibility complex class II and co-stimulatory molecules are present in human BAL fluid. Eur Respir J. 2003 Oct; 22(4):578-83. 